\title{
Przedsiębiorczość w rozwoju układów przestrzennych
}

\author{
Entrepreneurship \\ in the development \\ of spatial systems
}

